\title{
NONPARAMETRIC ESTIMATION OF EFFECTIVE DOSES AT QUANTAL RESPONSE
}

\author{
M.S. TIKHOV
}

\begin{abstract}
For the quantal response model we propose a new direct method for nonparametric estimation of the effective dose level $E D_{100 \lambda}(0<\lambda<1)$. This method yields a simple and reliable monotone estimate of the effective dose level curve $\lambda \rightarrow E D_{100 \lambda}$ and is appealing to users of conventional smoothing methods of kernel estimates. Moreover, it is computationally very efficient, because it does not require a numerical inversion of the estimate of the quantile dose response curve. We prove asymptotic normality of this new estimator and compare it with the DNP-estimator.
\end{abstract}

Keywords: binary response model, effective dose level, nonparametric estimate.

Mathematics Subject Classification: 62G05, 62G08, 62G20, $62 \mathrm{P} 10$.

\section{INTRODUCTION}

We consider the model of binary response which has a conventional title dose-response relationship [1] and which can be described as follows.

Let $\left\{\left(X_{i}, U_{i}\right), 1 \leqslant i \leqslant n\right\}$ be a potential repeated sample of an unknown distribution $F(x) Q(y), F(x)=\mathbf{P}\left(X_{i}<x\right), Q(y)=\mathbf{P}\left(U_{i}<y\right), x, y \in \mathbf{R}$, instead of which one observes the sample $\mathcal{U}^{(n)}=\left\{\left(U_{i}, W_{i}\right), 1 \leqslant i \leqslant n\right\}$, where $W_{i}=\chi\left(X_{i}<U_{i}\right)$ are the indicator functions of the event $\left(X_{i}<U_{i}\right)$. Here $U_{i}$ are regarded as injected doses, and $W_{i}$ as an effect of the action of the dose $U_{i}$. Let $F(x)=\int_{-\infty}^{x} f(t) d t$ and $f(x)>0$. We shall call this situation the random plan of an experiment.

Together with the random plan, we consider fixed plans of an experiment. Namely, the injected dose $U$ is supposed to be non-random and we let $U_{i}=u_{i}, i=0,1, \ldots, n+1$, where $0=u_{0}<u_{1}<\ldots<u_{n}<u_{n+1}=1$.

On the main problem of the dose-response relationship is to estimate the effective doses $E D_{100 \lambda}=F^{-1}(\lambda)=x_{\lambda}, 0<\lambda<1$, by the sample $\mathcal{U}^{(n)}$. For fixed plans of an experiment, we shall consider several nonparametric estimator and we shall find their asymptotic (as $n \rightarrow \infty)$ distributions.

The nonparametric approach to the estimating supposes the presence of kernel functions $K_{r}(x), K_{d}(x)$, being in fact even compactly supported densities of distributions with the support on $[-1,1]$, and bandwidth $h_{r}, h_{d}$, which are smoothing non-random parameters depending on the sampling size $n$ and converging to zero as $n \rightarrow \infty$, but $n h_{r} \rightarrow \infty, n h_{d} \rightarrow \infty$ as $n \rightarrow \infty$. We also let $H_{d}(u)=\int_{-\infty}^{u} K_{d}(x) d x$.

To estimate the function $F(x)$, we shall make use of the following statistics,

$$
F_{n h_{r}}(x)=\frac{1}{n h_{r}} \sum_{i=1}^{n} K_{r}\left(\frac{x-u_{i}}{h_{r}}\right) W_{i} .
$$

M.S. TikHov, Nonparametric eStimation of the EFfective doses at QUANTAl RESPonse.

(C) Tikhov M.S. 2013.

Submitted February 16, 2012. 
For fixed plans of an experiment in the dose-response relationship, in the present work we prove the asymptotic normality of the estimator

$$
\begin{aligned}
\hat{x}_{1, \lambda}=\frac{1}{n h_{d}} \sum_{i=1}^{n} \int_{-\infty}^{\lambda} K_{d} & \left(\frac{F_{n h_{r}}(i / n)-u}{h_{d}}\right) d u= \\
& =\frac{1}{n} \sum_{i=1}^{n} H_{d}\left(\frac{\lambda-F_{n h_{r}}(i / n)}{h_{d}}\right)
\end{aligned}
$$

for the effective dose $x_{\lambda}$ that we call the DNP-estimator.

We shall also study the asymptotic behavior of the estimator

$$
\begin{array}{r}
\hat{x}_{2, \lambda}=\frac{\sum_{i=1}^{n} \frac{2 i}{n} \int_{-\infty}^{\lambda} K_{d}\left(\frac{F_{n h_{r}}(i / n)-u}{h_{d}}\right) d u}{\sum_{i=1}^{n} \int_{-\infty}^{\lambda} K_{d}\left(\frac{u-F_{n h_{r}}(i / n)}{h_{d}}\right) d u}= \\
=\frac{\sum_{i=1}^{n} \frac{2 i}{n} H_{d}\left(\frac{\lambda-F_{n h_{r}}(i / n)}{h_{d}}\right)}{\sum_{i=1}^{n} H_{d}\left(\frac{\lambda-F_{n h_{r}}(i / n)}{h_{d}}\right)}
\end{array}
$$

for $x_{\lambda}$ that was suggested in work [2]. We show that the estimator $\hat{x}_{2, \lambda}$ has the same limiting distribution as the estimator $\hat{x}_{1, \lambda}$.

We also consider the asymptotic behavior of the estimator

$$
\hat{x}_{3, \lambda}=\sqrt{\hat{S}_{2, \lambda}-b\left(h_{r}, h_{d}\right)},
$$

where

$$
\hat{S}_{2, \lambda}=\frac{1}{n h_{d}} \sum_{i=1}^{n} \frac{2 i}{n} \int_{-\infty}^{\lambda} K_{d}\left(\frac{F_{n h_{r}}(i / n)-u}{h_{d}}\right) d u=\frac{1}{n} \sum_{i=1}^{n} \frac{2 i}{n} H_{d}\left(\frac{\lambda-F_{n h_{r}}(i / n)}{h_{d}}\right),
$$

and $b\left(h_{r}, h_{d}\right)$ are some constants depending on $h_{r}, h_{d}$ (see Theorem 4.1 ). We prove that the estimator $\hat{x}_{3, \lambda}$ is a consistent estimator for $x_{\lambda}$ and its limiting dispersion is less than the limiting dispersion of the estimator $\hat{x}_{1, \lambda}, \hat{x}_{2, \lambda}$.

We observe that in work [3], there was considered the regression model

$$
Y_{i}=m\left(X_{i}\right)+\sigma\left(X_{i}\right) \varepsilon_{i}, \quad i=1,2, \ldots, n,
$$

where $\left\{X_{i}, Y_{i}\right\}_{i=1}^{n}$ is a two-dimensional sample of independent identically distributed random variables, at that, a random variable $X_{i}$ has a density of distribution $f(x)>0$ and its values are located in the segment $[0,1]$, the random variables $\varepsilon_{i}$ are also supposed to be independent and identically distributed with expectation 0 and to have the fourth moment (and $\left\{\varepsilon_{i}\right\}_{i=1}^{n}$ are independent of $\left.\left\{X_{i}\right\}_{i=1}^{n}\right)$, while the regression function $m(x)$ is supposed to be strictly monotonous. The estimator $m_{I}^{-1}(\lambda)$ of the form (1) for the function $m^{-1}(\lambda)$ was suggested. It was also shown that the estimator $m_{I}^{-1}(\lambda)$ is asymptotically normal. To prove the asymptotic normality of the estimator $m_{I}^{-1}(\lambda)$, in [3] the independence of the variables $\left\{\varepsilon_{i}\right\}_{i=1}^{n}$ was employed essentially. In the relationship dose-response, the variables $W_{i}$ are binary quantities and therefore we can not employ representation (3). To prove the asymptotic normality, one needs to use another approach. 


\section{MAIN ASSUMPTIONS}

Let $\left\{X_{i}, i=1, \ldots, n\right\}$ be a sequence of independent identically distributed as $X$ on the segment $[0,1]$ random variables with the distribution function $F(x), P=\left\{u_{0}, u_{1}, \ldots, u_{n}, u_{n+1}\right\}$ be an ordered partition of the segment $[0,1], u_{0}=0<u_{1}<\ldots<u_{n}<1=u_{n+1}$.

We formulate the assumptions for the parameters $h_{r}$ and $h_{d}$.

\section{Assumptions (H).}

$\left(\mathbf{H}_{1}\right) \quad h_{r}=h_{r}(n), h_{d}=h_{d}(n)$, and $h_{r} \underset{n \rightarrow \infty}{\longrightarrow} 0, h_{d} \underset{n \rightarrow \infty}{\longrightarrow} 0$, but $n h_{r} \rightarrow \infty, n h_{d} \rightarrow \infty$ as $n \rightarrow \infty$.

$\left(\mathbf{H}_{2}\right) \quad h_{d} / h_{r} \underset{n \rightarrow \infty}{\longrightarrow} 0$.

$\left(\mathbf{H}_{3}\right) \quad n h_{r}^{5}=O(1)$ as $n \rightarrow \infty$.

$\left(\mathbf{H}_{4}\right) \quad n h_{r} h_{d}^{8 / 3} \underset{n \rightarrow \infty}{\longrightarrow} \infty$.

As an example, we consider $h_{r}=n^{-1 / 5}, h_{d}=n^{-1 / 4}$. It is obvious that these sequences satisfy Assumptions $(\mathbf{H})$.

We let $\|K\|^{2}=\int_{-1}^{1} K^{2}(x) d x$.

Assumptions for the kernel functions $K_{r}(x)$ and $K_{d}(x)$.

Assumptions (K).

$\left(\mathbf{K}_{1}\right) \quad K_{r(d)}(x) \geq 0$, and $K_{r(d)}(x)=0, x \notin[-1,1]$.

$\left(\mathbf{K}_{2}\right) \quad \int_{-1}^{1} K_{r}(x) d x=1, \quad \int_{-1}^{1} K_{d}(x) d x=1$.

$\left(\mathbf{K}_{3}\right) \quad K_{r(d)}(x)=K_{r(d)}(-x), x \in \mathbf{R}$.

$\left(\mathbf{K}_{4}\right)$ On the segment $[-1,1]$, there exist continuous bounded derivatives of the functions $K_{r}(x), K_{d}(x)$.

$\left(\mathbf{K}_{5}\right) \quad\left\|K_{j}\right\|_{\infty}=\sup _{x \in \mathbf{R}}\left|K_{j}(x)\right|=k_{j}<\infty$ for $j=r, d$.

Remark 2.1. Under Assumptions (K), there exist the fourth moments for the distributions with the densities $K_{r}(x), K_{d}(x)$ and

$$
\begin{aligned}
\nu_{r}^{2} & =\int x^{2} K_{r}(x) d x, & \nu_{d}^{2} & =\int x^{2} K_{d}(x) d x, \\
\mu_{r}^{4} & =\int x^{4} K_{r}(x) d x, & \mu_{d}^{4} & =\int x^{4} K_{d}(x) d x .
\end{aligned}
$$

Let us define the variation of the function $K$ (cf. [4, p. 234]).

Let $K:[a, b] \rightarrow \mathbf{R}$. The variation of the function $K=K(u)$ on the segment $[a, b]$ is the following quantity, $\bigvee(K)=\bigvee_{a}^{b}(K)=\sup _{P} \sum_{k=0}^{m}\left|K\left(u_{k+1}\right)-K\left(u_{k}\right)\right|$, i.e., the supremum over all ordered partitions $P$ of the segment $[a, b]$. Throughout the work we consider the variations of the functions on the segment $[0,1]$.

Remark 2.2. The boundedness of the derivatives of the functions $K_{r}(x), K_{d}(x)$ on the segment $[-1,1]$ (Assumption $\boldsymbol{K}_{4}$ ) imply that their derivatives are bounded (cf. [4, p. 235]), i.e., $\bigvee\left(K_{d(r)}\right)<\infty$. 


\section{Assumption (F).}

$\left(\mathbf{F}_{1}\right)$ There exists the third continuous bounded derivative of the density of the distribution $f(x)=F^{\prime}(x)$ and $f(x) \geq C_{0}>0$ for $0 \leqslant x \leqslant 1$, i.e., on the segment $[0,1]$, the density $f(x)$ is separated from zero.

\section{Assumption (P).}

$\left(\mathbf{P}_{1}\right) \quad$ As $n \rightarrow \infty$,

$$
\max _{k=0,1, \ldots, n} \max \left\{\left|u_{k}-\frac{k}{n}\right|,\left|u_{k+1}-\frac{k}{n}\right|\right\}=O\left(\frac{1}{n}\right) .
$$

Assumption $(\mathbf{P})$ yields $u_{k}=\frac{k}{n}+O\left(\frac{1}{n}\right)$, at that, the sequence $n\left(u_{k}-\frac{k}{n}\right)$ is bounded by a constant $C$ uniformly in $0 \leqslant k \leqslant n$.

Throughout the work (Main) Assumptions $(\mathbf{H}),(\mathbf{K}),(\mathbf{F}),(\mathbf{P})$ are supposed to hold true.

\section{AuXiLiary ReSUlts}

In this section we provide auxiliary results needed to study the asymptotics for the aforementioned estimators $\hat{x}_{1, \lambda}, \hat{x}_{2, \lambda}, \hat{x}_{3, \lambda}$.

We give first the Koksma-Hlawka inequality (see [5, p. 18]) that allows one to estimate the rate of the convergence of integral sums to the corresponding integral.

Let $\mathcal{B}$ be the Lebesgue $\sigma$-algebra on $I=[0,1]$ and $\rho$ is the Lebesgue measure on $\mathcal{B}$. For $P=\left\{u_{0}, u_{1}, \ldots, u_{n}, u_{n+1}\right\}$ with $u_{0}=0<u_{1}<\ldots<u_{n}<1=u_{n+1}$ and $B \in \mathcal{B}$ we define

$$
A(B ; P)=\sum_{i=1}^{n} \chi_{B}\left(u_{i}\right), \quad D_{n}(\mathcal{B} ; P)=\sup _{B \in \mathcal{B}}\left|\frac{A(B ; P)}{n}-\rho(B)\right|,
$$

where $\chi_{B}(x)$ is the indicator function for the set $B$. We let $D_{n}^{*}(P)=D_{n}\left(J_{c}^{*}, P\right)$, where $J_{c}^{*}$ is a subset of $I$ of the form $\left[0, u_{i}\right]$.

For each bounded function $\psi: \mathbf{R} \rightarrow \mathbf{R}$ we let $\|\psi\|_{I}=\sup _{x \in I}|\psi(x)|$.

Theorem 3.1 ([5], Koksma-Hlawka inequality). If a function $f(u)(0 \leqslant u \leqslant 1)$ has a bounded variation $\bigvee(f)$ on $[0,1]$, then for each $0<u_{1}<u_{2}<\ldots<u_{n}<1$ we have

$$
\left|\frac{1}{n} \sum_{i=1}^{n} f\left(u_{i}\right)-\int_{0}^{1} f(u) d u\right| \leqslant \bigvee(f) D_{n}^{*}\left(u_{1}, \ldots, u_{n}\right) .
$$

We give also two lemmata from [5].

Lemma 3.1. If $x_{1}, \ldots, x_{n}, y_{1}, \ldots, y_{n} \in[0,1]$ satisfy the inequalities $\left|x_{i}-y_{i}\right| \leqslant \varepsilon$ for $1 \leqslant$ $i \leqslant n$, then

$$
\left|D_{n}^{*}\left(x_{1}, \ldots, x_{n}\right)-D_{n}^{*}\left(y_{1}, \ldots, y_{n}\right)\right| \leqslant \varepsilon .
$$

Remark 3.1. Lemma 3.2 yields that $D_{n}^{*}\left(x_{1}, \ldots, x_{n}\right)$ is a continuous function of the variables $\left(x_{1}, \ldots, x_{n}\right)$.

Lemma 3.2. If $0<u_{1}<u_{2}<\ldots<u_{n}<1$, then

$$
D_{n}^{*}\left(u_{1}, \ldots, u_{n}\right)=\frac{1}{2 n}+\max _{1 \leqslant i \leqslant n}\left|u_{i}-\frac{2 i-1}{2 n}\right| .
$$

Remark 3.2. If $u_{i}=\frac{i}{n}$, then $\frac{i}{n}-\frac{2 i-1}{2 n}=\frac{1}{2 n}$ and $D_{n}^{*}\left(u_{1}, \ldots, u_{n}\right)=\frac{1}{n}$. 
Theorem 3.2 ([6, p. 337], [7, p. 299]). If $\varphi(n) \rightarrow \infty$ as $n \rightarrow \infty$ and

$$
\varphi(n)\left(T_{n}-\theta\right) \underset{n \rightarrow \infty}{\stackrel{d}{\longrightarrow}} N\left(0, \tau^{2}\right)
$$

then

$$
\varphi(n)\left(g\left(T_{n}\right)-g(\theta)\right) \underset{n \rightarrow \infty}{\stackrel{d}{\longrightarrow}} N\left(0, \tau^{2}\left(g^{\prime}(\theta)\right)^{2}\right)
$$

provided $g^{\prime}(\theta)$ exists and is not zero.

In what follows we shall make use of the following auxiliary result.

We consider the function

$$
\tilde{f}=\tilde{f}(u)=\frac{1}{h_{d}} K_{d}\left(\frac{F(u)-\lambda}{h_{d}}\right)
$$

and let us estimate it variation on $[0,1]$.

Lemma 3.3. If Main Assumptions hold true, then

$$
\bigvee(\tilde{f})=\sup \sum_{j=1}^{l}\left|\tilde{f}\left(u_{j}\right)-\tilde{f}\left(u_{j-1}\right)\right|=O\left(\frac{1}{h_{d}}\right)
$$

where the supremum is taken over all ordered partitions $0<u_{1}<u_{2}<\ldots<u_{l}<1$ of the segment $[0,1]$.

Proof. Let $0<u_{1}<u_{2}<\ldots<u_{l}<1$ be an arbitrary ordered partition of the segment $[0,1]$. Then

$$
\begin{gathered}
\sum_{j=1}^{l}\left|\tilde{f}\left(u_{j}\right)-\tilde{f}\left(u_{j-1}\right)\right|=\frac{1}{h_{d}} \sum_{j=1}^{l}\left|K_{d}\left(\frac{F\left(u_{j}\right)-\lambda}{h_{d}}\right)-K_{d}\left(\frac{F\left(u_{j-1}\right)-\lambda}{h_{d}}\right)\right|= \\
=\frac{1}{h_{d}}\left\{\sum_{j=1}^{l_{1}}+\sum_{j=l_{2}+2}^{l}+\sum_{j=l_{1}+2}^{l_{2}}\right\}\left|K_{d}\left(\frac{F\left(u_{j}\right)-\lambda}{h_{d}}\right)-K_{d}\left(\frac{F\left(u_{j-1}\right)-\lambda}{h_{d}}\right)\right|+ \\
+\frac{1}{h_{d}}\left|K_{d}\left(\frac{F\left(u_{l_{1}+1}\right)-\lambda}{h_{d}}\right)-K_{d}\left(\frac{F\left(u_{l_{1}}\right)-\lambda}{h_{d}}\right)\right|+ \\
+\frac{1}{h_{d}}\left|K_{d}\left(\frac{F\left(u_{l_{2}+1}\right)-\lambda}{h_{d}}\right)-K_{d}\left(\frac{F\left(u_{l_{2}+2}\right)-\lambda}{h_{d}}\right)\right|,
\end{gathered}
$$

where $l_{1}$ and $l_{2}$ are so that

$$
\begin{gathered}
F\left(u_{l_{1}}\right) \leqslant \lambda-h_{d}, \quad F\left(u_{l_{1}+1}\right)>\lambda-h_{d}, \\
F\left(u_{l_{2}+1}\right)<\lambda+h_{d}, \quad F\left(u_{l_{2}+2}\right) \geq \lambda+h_{d} .
\end{gathered}
$$

Since $K_{d}(x)=0$ for $|x| \geq 1$, the sum $\sum_{j=1}^{l_{1}}+\sum_{j=l_{2}+2}^{l}$ vanishes and

$$
K_{d}\left(\frac{F\left(u_{l_{1}+1}\right)-\lambda}{h_{d}}\right)=K_{d}(-1)+K_{d}^{\prime}(\xi)\left(\frac{F\left(u_{l_{1}+1}\right)-\lambda}{h_{d}}+1\right) \underset{n \rightarrow \infty}{\longrightarrow} 0,
$$

where $-1 \leqslant \xi \leqslant \frac{F\left(u_{l_{1}+1}\right)-\lambda}{h_{d}}$.

In the same way one can show that $K_{d}\left(\frac{F\left(u_{l_{2}}\right)-\lambda}{h_{d}}\right) \underset{n \rightarrow \infty}{\longrightarrow} 0$.

All the points $\frac{F\left(u_{j}\right)-\lambda}{h_{d}}$ in the remaining sum belongs to the segment $[-1,1]$ and hence

$$
\frac{1}{h_{d}} \sum_{j=l_{1}+2}^{l_{2}}\left|K_{d}\left(\frac{F\left(u_{j}\right)-\lambda}{h_{d}}\right)-K_{d}\left(\frac{F\left(u_{j-1}\right)-\lambda}{h_{d}}\right)\right|=
$$




$$
\begin{gathered}
=\frac{1}{h_{d}} \sum_{j=l_{1}+2}^{l_{2}}\left|K_{d}^{\prime}\left(\xi_{j}\right)\right| \frac{F\left(u_{j}\right)-F\left(u_{j-1}\right)}{h_{d}} \leqslant \frac{M}{h_{d}^{2}}\left(F\left(u_{l_{2}}\right)-F\left(u_{l_{1}+2}\right)\right) \leqslant \\
\leqslant \frac{2 M h_{d}}{h_{d}^{2}}=\frac{2 M}{h_{d}},
\end{gathered}
$$

where $\xi_{j} \in[-1,1],\left|K_{d}^{\prime}\left(\xi_{j}\right)\right| \leqslant M$ and $M$ is independent of $n$. The proof is complete.

\section{MAIn RESUltS}

4.1. Asymptotics for estimator $\hat{x}_{1, \lambda}$. We represent the statistics $\hat{x}_{1, \lambda}$ as

$$
\hat{x}_{1, \lambda}=\frac{1}{n h_{d}} \sum_{i=1}^{n} \int_{-\infty}^{\lambda} K_{d}\left(\frac{F_{n h_{r}}(i / n)-u}{h_{d}}\right) d u=x_{\lambda, n}+\Delta
$$

where

$$
\begin{gathered}
x_{\lambda, n}=\frac{1}{n h_{d}} \sum_{i=1}^{n} \int_{-\infty}^{\lambda} K_{d}\left(\frac{F(i / n)-u}{h_{d}}\right) d u \\
\Delta=\frac{1}{n h_{d}} \sum_{i=1}^{n} \int_{-\infty}^{\lambda}\left\{K_{d}\left(\frac{F_{n h_{r}}(i / n)-u}{h_{d}}\right)-K_{d}\left(\frac{F(i / n)-u}{h_{d}}\right)\right\} d u= \\
=\frac{1}{n} \sum_{i=1}^{n}\left(H_{d}\left(\frac{F_{n h_{r}}(i / n)-u}{h_{d}}\right)-H_{d}\left(\frac{F(i / n)-u}{h_{d}}\right)\right) .
\end{gathered}
$$

The asymptotic behavior of $x_{\lambda, n}$ is described in the following lemma.

Lemma 4.1. As $n \rightarrow \infty$,

$$
x_{\lambda, n}=x_{\lambda}+a_{2, d} h_{d}^{2}+o\left(h_{d}^{2}\right)
$$

where

$$
x_{\lambda}=F^{-1}(\lambda), \quad a_{2, d}=\frac{1}{2}\left(F^{-1}\right)^{\prime \prime}(\lambda) \nu_{d}^{2}=-\frac{\nu_{d}^{2} f^{\prime}\left(x_{\lambda}\right)}{2 f^{3}\left(x_{\lambda}\right)} .
$$

Proof. Employing the Koksma-Hlawka inequality, Lemma 3.4, and Remark 3.2, we obtain

$$
\begin{gathered}
x_{\lambda, n}=\frac{1}{h_{d}} \int_{0}^{1} \int_{-\infty}^{\lambda} K_{d}\left(\frac{F(x)-u}{h_{d}}\right) d u d x+O\left(\frac{1}{n h_{d}}\right)= \\
=\int_{0}^{1} d x \int_{\frac{F(x)-\lambda}{h_{d}}}^{1} K_{d}(z) d z+O\left(\frac{1}{n h_{d}}\right) .
\end{gathered}
$$

Since $\frac{F(x)-\lambda}{h_{d}} \leqslant-1$ as $x \leqslant F^{-1}\left(\lambda-h_{d}\right) \leqslant 1$, then

$$
x_{\lambda, n}=\int_{0}^{F^{-1}\left(\lambda-h_{d}\right)} d x \int_{-1}^{1} K_{d}(z) d z+\int_{F^{-1}\left(\lambda-h_{d}\right)}^{1} d x \int_{\frac{F(x)-\lambda}{h_{d}}}^{1} K_{d}(z) d z+O\left(\frac{1}{n h_{d}}\right) .
$$


The first integral is equal to $F^{-1}\left(\lambda-h_{d}\right)$, while in the second we make the change $y=\frac{F(x)-\lambda}{h_{d}}$ and, bearing in mind that $\lambda<F(1)=1, F \in C^{2}, f(x) \geq C_{0}>0$, we obtain

$$
\begin{gathered}
x_{\lambda, n}=F^{-1}\left(\lambda-h_{d}\right)+h_{d} \int_{-1}^{\frac{F(1)-\lambda}{h_{d}}} d y \int_{y}^{1} K_{d}(z)\left(F^{-1}\right)^{\prime}\left(\lambda+h_{d} y\right) d z+O\left(\frac{1}{n h_{d}}\right)= \\
=F^{-1}\left(\lambda-h_{d}\right)+h_{d} \int_{-1}^{1} d y \int_{y}^{1} K_{d}(z)\left\{\left(F^{-1}\right)^{\prime}(\lambda)+\left(F^{-1}\right)^{\prime \prime}(\lambda) y h_{d}+O\left(h_{d}^{2}\right)\right\} d z+O\left(\frac{1}{n h_{d}}\right) .
\end{gathered}
$$

Since

$$
\begin{gathered}
\int_{-1}^{1} d y \int_{y}^{1} K_{d}(z) d z=1, \quad \int_{-1}^{1} y d y \int_{y}^{1} K_{d}(z) d z=\frac{1}{2} \nu_{d}^{2}-\frac{1}{2}, \\
\sup _{t, x \in[0,1]}\left|\left(F^{-1}\right)^{\prime}(t)-\left(F^{-1}\right)^{\prime}(x)-(t-x)\left(F^{-1}\right)^{\prime \prime}(x)\right| \leqslant \frac{1}{2} \sup _{x \in[0,1]}\left|\left(F^{-1}\right)^{\prime \prime \prime}(x)\right|,
\end{gathered}
$$

and

$$
\left(F^{-1}\right)^{\prime \prime \prime}(x)=\frac{3\left(f^{\prime}\left(F^{-1}(x)\right)\right)^{2}}{\left(f\left(F^{-1}(x)\right)\right)^{5}}-\frac{f^{\prime \prime}\left(F^{-1}(x)\right)}{\left(f\left(F^{-1}(x)\right)\right)^{4}},
$$

by the separation of the density from the zero and the boundedness of the derivatives for the density of distribution we obtain that

$$
\sup _{t, x \in[0,1]}\left|\left(F^{-1}\right)^{\prime}(t)-\left(F^{-1}\right)^{\prime}(x)-(t-x)\left(F^{-1}\right)^{\prime \prime}(x)\right| \leqslant C .
$$

Thus,

$$
\begin{gathered}
x_{\lambda, n}=F^{-1}\left(\lambda-h_{d}\right)+ \\
+h_{d}\left(\left(F^{-1}\right)^{\prime}(\lambda)+\left(F^{-1}\right)^{\prime \prime}(\lambda) h_{d} \int_{-1}^{1} y d y \int_{y}^{1} K_{d}(z) d z+O\left(h_{d}^{2}\right)\right)+O\left(\frac{1}{n h_{d}}\right)= \\
=F^{-1}(\lambda)+\frac{1}{2} h_{d}^{2}\left(F^{-1}\right)^{\prime \prime}(\lambda) \nu_{d}^{2}+O\left(h_{d}^{3}+\frac{1}{n h_{d}}\right),
\end{gathered}
$$

that completes the proof.

Consider the variable $\Delta$ and represent it as

$$
\Delta=\Delta_{1}+\frac{1}{2} \Delta_{2}+\frac{1}{6} \Delta_{3} .
$$

Here

$$
\begin{gathered}
\Delta_{1}=-\frac{1}{n h_{d}} \sum_{i=1}^{n} K_{d}\left(\frac{\lambda-F(i / n)}{h_{d}}\right)\left(F_{n h_{r}}(i / n)-F(i / n)\right), \\
\Delta_{2}=\frac{1}{n h_{d}^{2}} \sum_{i=1}^{n} K_{d}^{\prime}\left(\frac{\lambda-F(i / n)}{h_{d}}\right)\left(F_{n h_{r}}(i / n)-F(i / n)\right)^{2}, \\
\Delta_{3}=-\frac{1}{n h_{d}^{3}} \sum_{i=1}^{n} K_{d}^{\prime \prime}\left(\frac{\lambda-\xi_{i}}{h_{d}}\right)\left(F_{n h_{r}}(i / n)-F(i / n)\right)^{3}
\end{gathered}
$$

where $\left|\xi_{i}-F(i / n)\right| \leqslant\left|F(i / n)-F_{n h_{r}}(i / n)\right|$. 
Lemma 4.2. As $n \rightarrow \infty$,

$$
\sqrt{n h_{r}}\left(\Delta_{1}-a_{2, r} h_{d}^{2}\right) \stackrel{d}{\rightarrow} N\left(0, g_{2}^{2}\right)
$$

where

$$
\begin{gathered}
a_{2, r}=-\frac{\nu_{r}^{2}}{2} F^{\prime \prime}\left(F^{-1}(\lambda)\right)\left(F^{-1}\right)^{\prime}(\lambda)=-\frac{\nu_{r}^{2} f^{\prime}\left(x_{\lambda}\right)}{2 f\left(x_{\lambda}\right)}, \\
g_{2}^{2}=\lambda(1-\lambda)\left\|K_{r}\right\|^{2}\left[\left(F^{-1}\right)^{\prime}(\lambda)\right]^{2}=\frac{\lambda(1-\lambda)}{f^{2}\left(x_{\lambda}\right)}\left\|K_{r}\right\|^{2} .
\end{gathered}
$$

Proof. We define the variables

$$
\begin{gathered}
\Delta_{1,1}=-\frac{1}{n h_{d}} \sum_{i=1}^{n} K_{d}\left(\frac{F(i / n)-\lambda}{h_{d}}\right)\left(F_{n h_{r}}(i / n)-\mathbf{E}\left(F_{n h_{r}}(i / n)\right)\right), \\
\Delta_{1,2}=-\frac{1}{n h_{d}} \sum_{i=1}^{n} K_{d}\left(\frac{F(i / n)-\lambda}{h_{d}}\right)\left(\mathbf{E}\left(F_{n h_{r}}(i / n)\right)-F(i / n)\right) .
\end{gathered}
$$

Then $\Delta_{1}=\Delta_{1,1}+\Delta_{1,2}$, and $\Delta_{1,2}$ is non-random.

It follows from [8, p. 68] that

$$
\sup _{x}\left|\mathbf{E}\left(F_{n h_{r}}(x)-F(x)\right)\right| \leqslant \frac{1}{2} h_{r}^{2} \nu_{r}^{2} \sup _{x}\left|f^{\prime}(x)\right| \leqslant \frac{M_{1} h_{r}^{2} \nu_{r}^{2}}{2} .
$$

Employing this fact, we obtain

$$
\begin{gathered}
\mathbf{E}\left(\Delta_{1}\right)=\mathbf{E}\left(\Delta_{1,2}\right)=-\frac{\nu_{r}^{2} h_{r}^{2}}{2 n h_{d}} \sum_{i=1}^{n} K_{d}\left(\frac{F(i / n)-\lambda}{h_{d}}\right) F^{\prime \prime}(i / n)(1+o(1))= \\
=-\frac{\nu_{r}^{2} h_{r}^{2}}{2 h_{d}} \int_{0}^{1} K_{d}\left(\frac{F(x)-\lambda}{h_{d}}\right) F^{\prime \prime}(x) d x(1+o(1))+O\left(\frac{h_{r}^{2}}{n h_{d}}\right)= \\
=-\frac{\nu_{r}^{2} h_{r}^{2}}{2} \int_{-1}^{1} K_{d}(z)\left(F^{-1}\right)^{\prime}\left(\lambda+z h_{d}\right) F^{\prime \prime}\left(F^{-1}\left(\lambda+z h_{d}\right)\right) d z(1+o(1))+O\left(\frac{h_{r}^{2}}{n h_{d}}\right)= \\
=-\frac{\nu_{r}^{2}}{2} h_{r}^{2}\left(F^{-1}\right)^{\prime}(\lambda) F^{\prime \prime}\left(F^{-1}(\lambda)\right)+o\left(h_{r}^{2}\right) .
\end{gathered}
$$

Let us calculate the variance of $\Delta_{1}$. We have

$$
\begin{aligned}
& \quad \mathbf{D}\left(\Delta_{1}\right)=\mathbf{D}\left(\Delta_{1,1}\right)= \\
& =\frac{1}{n^{4} h_{d}^{2} h_{r}^{2}} \sum_{j=1}^{n} F\left(u_{j}\right)\left(1-F\left(u_{j}\right)\right)\left\{\sum_{i=1}^{n} K_{d}\left(\frac{F(i / n)-\lambda}{h_{d}}\right) K_{r}\left(\frac{i / n-u_{j}}{h_{r}}\right)\right\}^{2}= \\
& =\frac{1}{n^{2} h_{d}^{2} h_{r}^{2}} \sum_{j=1}^{n} F\left(u_{j}\right)\left(1-F\left(u_{j}\right)\right)\left\{\int_{0}^{1} K_{d}\left(\frac{F(x)-\lambda}{h_{d}}\right) K_{r}\left(\frac{x-u_{j}}{h_{r}}\right) d x+O\left(\frac{1}{n}\right)\right\}^{2} .
\end{aligned}
$$

We make the change $z=\frac{F(x)-\lambda}{h_{d}}$ and apply Koksma-Hlavka inequality. Then

$$
\mathbf{D}\left(\Delta_{1}\right)=\frac{1}{n h_{d}^{2} h_{r}^{2}} \int_{0}^{1} F(y)(1-F(y)) \times
$$




$$
\times\left\{\int_{0}^{1} K_{d}\left(\frac{F(x)-\lambda}{h_{d}}\right) K_{r}\left(\frac{x-y}{h_{r}}\right) d x+O\left(\frac{1}{n}\right)\right\}^{2} d y+O\left(\frac{1}{n^{2} h_{r}^{2}}\right) .
$$

Moreover, as $n \rightarrow \infty$

$$
K_{r}\left(\frac{F^{-1}\left(\lambda+h_{d} z\right)-y}{h_{r}}\right)=K_{r}\left(\frac{F^{-1}(\lambda)-y}{h_{r}}\right)+o(1) .
$$

Taking into consideration the latter and making the change $t=\frac{F^{-1}(\lambda)-y}{h_{r}}$, we finally get

$$
\mathbf{D}\left(\Delta_{1}\right)=\frac{\lambda(1-\lambda)\left\|K_{r}\right\|^{2}}{f^{2}\left(x_{\lambda}\right) n h_{r}}+o\left(\frac{1}{n h_{r}}\right) .
$$

Now, to prove the asymptotic normality of $\Delta_{1}$, it is sufficient to prove the asymptotic normality of $\Delta_{1,1}$. In order to do it, we represent $\Delta_{1,1}$ as the sum $\Delta_{1,1}=\sum_{i=1}^{n} \xi_{j}$, where

$$
\xi_{j}=-\frac{1}{n^{2} h_{d} h_{r}}\left(\chi\left(X_{j}<u_{j}\right)-F\left(u_{j}\right)\right) \sum_{i=1}^{n} K_{d}\left(\frac{F(i / n)-\lambda}{h_{d}}\right) K_{r}\left(\frac{i / n-u_{j}}{h_{r}}\right) .
$$

Let $G(u)=F(u)-4 F^{2}(u)+6 F^{3}(u)-3 F^{4}(u)$. Then

$$
\begin{gathered}
\sum_{j=1}^{n} \mathbf{E}\left(\xi_{j}-\mathbf{E}\left(\xi_{j}\right)\right)^{4}=\sum_{j=1}^{n} \mathbf{E}\left(\xi_{j}\right)^{4}=\frac{1}{n^{8} h_{d}^{4} h_{r}^{4}} \sum_{j=1}^{n} \mathbf{E}\left(\chi\left(X_{j}<u_{j}\right)-F\left(u_{j}\right)\right)^{4} \times \\
\times\left\{\sum_{i=1}^{n} K_{d}\left(\frac{F(i / n)-\lambda}{h_{d}}\right) K_{r}\left(\frac{i / n-u_{j}}{h_{r}}\right)\right\}^{4}= \\
=\frac{1}{n^{8} h_{d}^{4} h_{r}^{4}} \sum_{j=1}^{n} G\left(u_{j}\right)\left\{\int_{0}^{1} K_{d}\left(\frac{F(x)-\lambda}{h_{d}}\right) K_{r}\left(\frac{x-u_{j}}{h_{r}}\right) d x+O\left(\frac{1}{n}\right)\right\}^{4}= \\
=\frac{1}{n^{4} h_{r}^{4}} \sum_{j=1}^{n} G\left(u_{j}\right)\left\{\int_{0}^{1} K_{d}(y) K_{r}\left(\frac{x-u_{j}}{h_{r}}\right) x_{y}^{\prime} d y+O\left(\frac{1}{n h_{d}}\right)\right\}^{4}= \\
=\frac{1}{n^{3} h_{r}^{3}} \int_{-1}^{1} G\left(x-z h_{r}\right)\left\{\int_{-1}^{1} K_{d}(y) K_{r}(z)\left(F^{-1}\right)^{\prime}\left(\lambda+h_{d} y\right) d y\right\}^{4} d z+O\left(\frac{1}{n^{4} h_{d}^{4}}\right)=O\left(\frac{1}{n^{3} h_{d}^{3}}\right) .
\end{gathered}
$$

Since

$$
\frac{\sum_{j=1}^{n} \mathbf{E}\left(\xi_{j}-\mathbf{E}\left(\xi_{j}\right)\right)^{4}}{\left(\mathbf{D}\left(\sum_{j=1}^{n} \xi_{j}\right)\right)^{2}}=\frac{\sum_{j=1}^{n} \mathbf{E}\left(\xi_{j}-\mathbf{E}\left(\xi_{j}\right)\right)^{4}}{\left(\mathbf{D}\left(\Delta_{1}\right)\right)^{2}}=O\left(\frac{1}{n h_{r}}\right) \underset{n \rightarrow \infty}{\longrightarrow} 0,
$$

the sequence $\sum_{j=1}^{n} \xi_{j}$ satisfies the assumptions of Lyapunov central limit theorem. This completes the proof.

Lemma 4.3. As $n \rightarrow \infty$,

$$
\Delta_{2}+\Delta_{3}=o\left(\frac{1}{\sqrt{n h_{r}}}\right)
$$


Proof. First we consider $\Delta_{2}$. We have

$$
\begin{gathered}
\left|\mathbf{E}\left(\Delta_{2}\right)\right| \leqslant \frac{1}{n h_{d}} \sum_{i=1}^{n}\left|\frac{1}{h_{d}} K_{d}^{\prime}\left(\frac{\lambda-F(i / n)}{h_{d}}\right)\right| \mathbf{E}\left(F_{n h_{r}}(i / n)-F(i / n)\right)^{2} \leqslant \\
\leqslant \frac{C_{1} h_{r}^{4}}{n h_{d}} \sum_{i=1}^{n}\left|\frac{1}{h_{d}} K_{d}^{\prime}\left(\frac{\lambda-F(i / n)}{h_{d}}\right)\right|= \\
=\frac{C_{1} h_{r}^{4}}{h_{d}} \int_{-1}^{1}\left|K_{d}^{\prime}(t)\right| d t+O\left(\frac{h_{r}^{4}}{n h_{d}^{2}}\right)= \\
=O\left(\frac{h_{r}^{4}}{h_{d}}\right)=o\left(\frac{1}{\sqrt{n h_{r}}}\right) .
\end{gathered}
$$

Then

$$
-\mathbf{E}\left(\Delta_{3}\right)=\frac{1}{n h_{d}^{3}} \sum_{i=1}^{n} K_{d}^{\prime \prime}\left(\frac{\lambda-\xi_{i}}{h_{d}}\right) \mathbf{E}\left(\left(F_{n h_{r}}(i / n)-F(i / n)\right)^{3}\right) .
$$

Let $A(x)=\mathbf{E}\left(\left(F_{n h_{r}}(x)-F(x)\right)^{3}\right)$, then

$$
\begin{gathered}
A(x)=\mathbf{E}\left(\left(F_{n h_{r}}(x)-\mathbf{E}\left(F_{n h_{r}}(x)\right)+\mathbf{E}\left(F_{n h_{r}}(x)\right)-F(x)\right)^{3}\right)= \\
\left.\left.=\mathbf{E}\left(\left(F_{n h_{r}}(x)-\mathbf{E}\left(F_{n h_{r}}(x)\right)\right)^{3}\right)+\left(\mathbf{E}\left(F_{n h_{r}}(x)\right)-F(x)\right)\right)^{3}+3 \mathbf{D}\left(F_{n h_{r}}\right) \cdot\left(\mathbf{E}\left(F_{n h_{r}}(x)\right)-F(x)\right)\right)= \\
=\mathbf{E}\left(\left(F_{n h_{r}}(x)-\mathbf{E}\left(F_{n h_{r}}(x)\right)\right)^{3}\right)+O\left(h_{r}^{6}+\frac{h_{r}}{n}\right),
\end{gathered}
$$

and these estimates are uniform in $x$ and thus

$$
\mid \mathbf{E}\left(\Delta_{3} \mid \leqslant \frac{M_{2}}{h_{d}^{3}} \int_{-1}^{1} A(x) d x .\right.
$$

Consider now

$$
\mathbf{E}\left(\left(F_{n h_{r}}(x)-\mathbf{E}\left(F_{n h_{r}}(x)\right)\right)^{3}\right)=\mathbf{E}\left(\left(n^{-1} \sum_{j=1}^{n} \eta_{j}(x)\right)^{3}\right),
$$

where

$$
\eta_{j}(x)=\frac{1}{h_{r}}\left(\chi\left(X_{j}<x\right)-F(x)\right) K_{r}\left(\frac{x-u}{h_{r}}\right) .
$$

Then (cf. [9, p. 379])

$$
\mathbf{E}\left(\left(n^{-1} \sum_{j=1}^{n} \eta_{j}(x)\right)^{3}\right)=n^{-2} \mathbf{E}\left(\eta_{1}^{3}(x)\right)=\frac{F(x)-3 F^{2}(x)+2 F^{3}(x)}{n^{2} h_{r}^{3}} K_{r}^{3}\left(\frac{x-u}{h_{r}}\right) .
$$

Employing the boundedness of $K_{d}^{\prime \prime}(t)$ and the fact that

$$
\frac{1}{h_{r}} \int_{-1}^{1} K_{r}^{3}\left(\frac{x-u}{h_{r}}\right) d x \leqslant M_{3}<\infty,
$$

we obtain

$$
\left|\mathbf{E}\left(\Delta_{3}\right)\right|=O\left(\frac{1}{n^{2} h_{d}^{3} h_{r}^{2}}\right)=o\left(\frac{1}{\sqrt{n h_{r}}}\right) .
$$

In the same one can show that $\mathbf{E}\left(\Delta_{2}^{2}\right), \mathbf{E}\left(\Delta_{3}^{2}\right)$ converge to zero as $n \rightarrow \infty$. Hence, by Chebyshev inequality, we complete the proof.

Lemmata 4.1-4.3 imply the following theorem. 
Theorem 4.1. As $n \rightarrow \infty$,

$$
\sqrt{n h_{r}}\left(\hat{x}_{1, \lambda}-x_{\lambda}-b_{2}\left(h_{r}, h_{d}\right)\right) \stackrel{d}{\rightarrow} N\left(0, g_{2}^{2}\right),
$$

where

$$
\begin{gathered}
b_{2}\left(h_{r}, h_{d}\right)=a_{2, d} h_{d}^{2}+a_{2, r} h_{r}^{2}, \quad a_{2, r}=-\frac{\nu_{r}^{2} f^{\prime}\left(x_{\lambda}\right)}{2 f\left(x_{\lambda}\right)}, \quad a_{2, d}=-\frac{\nu_{d}^{2} f^{\prime}\left(x_{\lambda}\right)}{2 f^{3}\left(x_{\lambda}\right)}, \\
g_{2}^{2}=\frac{\lambda(1-\lambda)\left\|K_{r}\right\|^{2}}{f^{2}\left(x_{\lambda}\right)} .
\end{gathered}
$$

4.2. Asymptotics for estimators $\hat{x}_{2, \lambda}$ and $\hat{x}_{3, \lambda}$. To study the asymptotics for the estimators $\hat{x}_{2, \lambda}$, we represent it as

where

$$
\hat{x}_{2, \lambda}=\frac{\hat{S}_{2, \lambda}}{\hat{x}_{1, \lambda}},
$$

$$
\begin{gathered}
\hat{S}_{2, \lambda}=x_{2, \lambda}+2 \Lambda, \quad x_{2, \lambda}=\frac{2}{n h_{d}} \sum_{i=1}^{n} \frac{i}{n} \int_{-\infty}^{\lambda} K_{d}\left(\frac{F(i / n)-u}{h_{d}}\right) d u \\
\Lambda=\frac{1}{n h_{d}} \sum_{i=1}^{n} \frac{i}{n} \int_{-\infty}^{\lambda}\left\{K_{d}\left(\frac{F_{n h_{r}}(i / n)-u}{h_{d}}\right)-K_{d}\left(\frac{F(i / n)-u}{h_{d}}\right)\right\} d u .
\end{gathered}
$$

Lemma 4.4. As $n \rightarrow \infty$,

$$
x_{2, \lambda}=x_{\lambda}^{2}+h_{d}^{2} \nu_{d}^{2} x_{\lambda}\left(-\frac{f^{\prime}\left(x_{\lambda}\right)}{f^{3}\left(x_{\lambda}\right)}+\frac{1}{f^{2}\left(x_{\lambda}\right)}\right)+o\left(h_{d}^{2}\right) .
$$

Proof. Applying the Koksma-Hlawka inequality, we obtain

$$
\begin{gathered}
x_{2, \lambda}=\frac{2}{h_{d}} \int_{0}^{1} \int_{-\infty}^{\lambda} x K_{d}\left(\frac{F(x)-u}{h_{d}}\right) d u d x+O\left(\frac{1}{n h_{d}}\right)= \\
=2 \int_{0}^{1} x d x \int_{(F(x)-\lambda) / h_{d}}^{1} K_{d}(y) d y+O\left(\frac{1}{n h_{d}}\right)= \\
=2 \int_{0}^{F^{-1}\left(\lambda-h_{d}\right)} x d x \int_{-1}^{1} K_{d}(y) d y+2 \int_{F^{-1}\left(\lambda-h_{d}\right)}^{1} x d x \int_{(F(x)-\lambda) / h_{d}}^{1} K_{d}(y) d y+O\left(\frac{1}{n h_{d}}\right) .
\end{gathered}
$$

The first integral can be immediately calculated, while in the other we make the change $t=\frac{F(x)-\lambda}{h_{d}}$. It yields

$$
\begin{gathered}
x_{2, \lambda}=\left(F^{-1}\left(\lambda-h_{d}\right)\right)^{2}+ \\
+2 h_{d} \int_{-1}^{(F(1)-\lambda) / h_{d}}\left(F^{-1}\right)^{\prime}\left(\lambda+t h_{d}\right) F^{-1}\left(\lambda+t h_{d}\right) d t \int_{t}^{1} K_{d}(y) d y+O\left(\frac{1}{n h_{d}}\right)= \\
=\left\{F^{-1}(\lambda)-\left(F^{-1}\right)^{\prime}(\lambda) h_{d}+\left(F^{-1}\right)^{\prime \prime}(\lambda) \frac{h_{d}^{2}}{2}+o\left(h_{d}^{2}\right)\right\}^{2}+ \\
\quad+2 h_{d}\left(F^{-1}\right)^{\prime}(\lambda) F^{-1}(\lambda) \int_{-1}^{1} d t \int_{t}^{1} K_{d}(y) d y+
\end{gathered}
$$




$$
+2 h_{d}^{2} \int_{-1}^{1} t d t \int_{t}^{1} K_{d}(y) d y\left\{\left(F^{-1}\right)^{\prime \prime}(\lambda) F^{-1}(\lambda)+\left[\left(F^{-1}\right)^{\prime}(\lambda)\right]^{2}\right\}+o\left(h_{d}^{2}\right) .
$$

Since

$$
\int_{-1}^{1} d t \int_{t}^{1} K_{d}(y) d y=1, \quad 2 \int_{-1}^{1} d t \int_{t}^{1} K_{d}(y) t d y=\nu_{d}^{2}-1
$$

then

$$
\begin{aligned}
x_{2, \lambda}=\left\{\left(F^{-1}(\lambda)\right)^{2}+\left(\left(F^{-1}\right)^{\prime}(\lambda)\right)^{2} h_{d}^{2}-2 F^{-1}(\lambda)\left(F^{-1}\right)^{\prime}(\lambda) h_{d}+\right. \\
\left.+F^{-1}(\lambda)\left(F^{-1}\right)^{\prime \prime}(\lambda) h_{d}^{2}\right\}+2\left(F^{-1}\right)^{\prime}(\lambda) F^{-1}(\lambda) h_{d}+ \\
+h_{d}^{2}\left(\nu_{d}^{2}-1\right) F^{-1}(\lambda)\left\{\left(F^{-1}\right)^{\prime \prime}(\lambda)+\left(\left(F^{-1}\right)^{\prime}(\lambda)\right)^{2}\right\}+o\left(h_{d}^{2}\right) .
\end{aligned}
$$

It completes the proof.

We represent the variable $\Lambda$ as the sum $\Lambda=\Lambda_{1}+\frac{1}{2} \Lambda_{2}+\frac{1}{6} \Lambda_{3}$, where

$$
\begin{gathered}
\Lambda_{1}=-\frac{1}{n h_{d}} \sum_{i=1}^{n} K_{d}\left(\frac{\lambda-F(i / n)}{h_{d}}\right) \frac{i}{n}\left(F_{n h_{r}}(i / n)-F(i / n)\right), \\
\Lambda_{2}=\frac{1}{n h_{d}^{2}} \sum_{i=1}^{n} K_{d}^{\prime}\left(\frac{F(i / n)-u}{h_{d}}\right) \frac{i}{n}\left(F_{n h_{r}}(i / n)-F(i / n)\right)^{2}, \\
\Lambda_{3}=-\frac{1}{n h_{d}^{3}} \sum_{i=1}^{n} K_{d}^{\prime \prime}\left(\frac{\xi_{i}-u}{h_{d}}\right) \frac{i}{n}\left(F_{n h_{r}}(i / n)-F(i / n)\right)^{3}, \\
\left|\xi_{i}-F(i / n)\right| \leqslant\left|F(i / n)-F_{n h_{r}}(i / n)\right| .
\end{gathered}
$$

Lemma 4.5. As $n \rightarrow \infty$,

$$
\sqrt{n h_{r}}\left(\Lambda_{1}-a_{1, r} h_{r}^{2}\right) \stackrel{d}{\rightarrow} N\left(0, g_{1}^{2}\right)
$$

where

$$
a_{1, r}=-\frac{\nu_{r}^{2} x_{\lambda} f^{\prime}\left(x_{\lambda}\right)}{f^{4}\left(x_{\lambda}\right)}, \quad g_{1}^{2}=\frac{4 \lambda(1-\lambda) x_{\lambda}^{2}}{f^{2}\left(x_{\lambda}\right)}\left\|K_{r}\right\|^{2} .
$$

Proof. Let

$$
\begin{gathered}
\Lambda_{1,1}=-\frac{1}{n h_{d}} \sum_{i=1}^{n} K_{d}\left(\frac{F(i / n)-\lambda}{h_{d}}\right) \frac{i}{n}\left(F_{n h_{r}}(i / n)-\mathbf{E}\left(F_{n h_{r}}(i / n)\right)\right), \\
\Lambda_{1,2}=-\frac{1}{n h_{d}} \sum_{i=1}^{n} K_{d}\left(\frac{F(i / n)-\lambda}{h_{d}}\right) \frac{i}{n}\left(\mathbf{E}\left(F_{n h_{r}}(i / n)\right)-F(i / n)\right) .
\end{gathered}
$$

Then $\Lambda_{1}=\Lambda_{1,1}+\Lambda_{1,2}$.

Taking into consideration that $\mathbf{E}\left(F_{n h_{r}}(x)-F(x)\right)=\frac{\nu_{r}^{2} h_{r}^{2}}{2} f^{\prime}(x)+o\left(h_{r}^{2}\right)$, we obtain

$$
\begin{gathered}
\mathbf{E}\left(\Lambda_{1}\right)=\mathbf{E}\left(\Lambda_{1,2}\right)=-\frac{\nu_{r}^{2} h_{r}^{2}}{2 n h_{d}} \sum_{i=1}^{n} K_{d}\left(\frac{F(i / n)-\lambda}{h_{d}}\right) \frac{i}{n} F^{\prime \prime}(i / n)(1+o(1))= \\
=-\frac{\nu_{r}^{2} h_{r}^{2}}{2 h_{d}} \int_{0}^{1} K_{d}\left(\frac{F(x)-\lambda}{h_{d}}\right) x F^{\prime \prime}(x) d x(1+o(1))+O\left(\frac{h_{r}^{2}}{n h_{d}}\right)=
\end{gathered}
$$

$=-\frac{\nu_{r}^{2} h_{r}^{2}}{2} \int_{-1}^{1} K_{d}(z) F^{-1}\left(\lambda+z h_{d}\right)\left(F^{-1}\right)^{\prime}\left(\lambda+z h_{d}\right) F^{\prime \prime}\left(F^{-1}\left(\lambda+z h_{d}\right)\right) d z(1+o(1))+O\left(\frac{h_{r}^{2}}{n h_{d}}\right)=$ 


$$
=-\frac{\nu_{r}^{2} h_{r}^{2}}{2} F^{-1}(\lambda)\left(F^{-1}\right)^{\prime}(\lambda) F^{\prime \prime}\left(F^{-1}(\lambda)\right)+o\left(h_{d}^{2}\right) .
$$

Let us calculate the variance of the variable $\Lambda_{1}$. We have

$$
\begin{aligned}
& \mathbf{D}\left(\Lambda_{1}\right)=\mathbf{D}\left(\Lambda_{1,1}\right)= \\
= & \frac{1}{n^{4} h_{d}^{2} h_{r}^{2}} \sum_{j=1}^{n} F\left(u_{j}\right)\left(1-F\left(u_{j}\right)\right)\left\{\sum_{i=1}^{n} K_{d}\left(\frac{F(i / n)-\lambda}{h_{d}}\right) K_{r}\left(\frac{i / n-u_{j}}{h_{r}}\right) \frac{i}{n}\right\}^{2}= \\
= & \frac{1}{n^{2} h_{d}^{2} h_{r}^{2}} \sum_{j=1}^{n} F\left(u_{j}\right)\left(1-F\left(u_{j}\right)\right)\left\{\int_{0}^{1} K_{d}\left(\frac{F(x)-\lambda}{h_{d}}\right) K_{r}\left(\frac{x-u_{j}}{h_{r}}\right) x d x+O\left(\frac{1}{n}\right)\right\}^{2} .
\end{aligned}
$$

Making the change $z=\frac{F(x)-\lambda}{h_{d}}$ and applying again Koksma-Hlawka inequality, we obtain

$$
\mathbf{D}\left(\Lambda_{1}\right)=\frac{1}{n h_{d}^{2} h_{r}^{2}} \int_{0}^{1} F(u)(1-F(u)) d u \times
$$

$\times\left\{\int_{0}^{1} K_{d}(z) K_{r}\left(\frac{F^{-1}\left(\lambda+z h_{d}\right)-u}{h_{r}}\right) F^{-1}\left(\lambda+z h_{d}\right)\left(F^{-1}\right)^{\prime}\left(\lambda+z h_{d}\right) d x+O\left(\frac{1}{n}\right)\right\}^{2}+O\left(\frac{1}{n^{2} h_{r}^{2}}\right)$.

Employing that, as $n \rightarrow \infty$,

$$
K_{r}\left(\frac{F^{-1}\left(\lambda+h_{d} z\right)-y}{h_{r}}\right)=K_{r}\left(\frac{F^{-1}(\lambda)-y}{h_{r}}\right)+o(1),
$$

and making the change $\frac{F^{-1}(\lambda)-u}{h_{r}}$, we finally get

$\mathbf{D}\left(\Lambda_{1}\right)=\frac{1}{n h_{r}} \lambda(1-\lambda)\left(F^{-1}(\lambda)\left(F^{-1}\right)^{\prime}(\lambda)\right)^{2}\left\|K_{r}\right\|^{2}+o\left(\frac{1}{n h_{r}}\right)=\frac{\lambda(1-\lambda) x_{\lambda}^{2}\left\|K_{r}\right\|^{2}}{n h_{r} f^{2}\left(x_{\lambda}\right)}+o\left(\frac{1}{n h_{r}}\right)$.

Taking into the fact 2 in the definition of the statistics $\hat{S}_{2, \lambda}$, we complete the proof.

Lemma 4.6. As $n \rightarrow \infty$,

$$
\Lambda_{2}+\Lambda_{3}=o\left(\frac{1}{\sqrt{n h_{r}}}\right) .
$$

Proof. Bearing in mind that $0 \leqslant i / n \leqslant 1$ and reproducing the proof of Lemma 4.3, we obtain the statement of the lemma.

Lemma 4.7. As $n \rightarrow \infty$,

$$
\sqrt{n h_{r}}\left(\hat{S}_{2, \lambda}-x_{\lambda}^{2}-a_{1}\left(h_{r}, h_{d}\right) h_{d}^{2}\right) \stackrel{d}{\rightarrow} N\left(0, g_{1}^{2}\right),
$$

where

$$
\begin{gathered}
g_{1}^{2}=\frac{\lambda(1-\lambda) x_{\lambda}^{2}}{f^{2}\left(x_{\lambda}\right)}\left\|K_{r}\right\|^{2} \\
a_{1, d}=\nu_{d}^{2}\left(-\frac{x_{\lambda} f^{\prime}\left(x_{\lambda}\right)}{f^{3}\left(x_{\lambda}\right)}+\frac{1}{f^{2}\left(x_{\lambda}\right)}\right) .
\end{gathered}
$$


The proof of this lemma follows the same lines as that of Lemma 4.4 and we omit it.

We represent the estimator $\hat{x}_{2, \lambda}$ as the fraction $\frac{\beta}{\alpha}$, where

$$
\beta=x_{2, \lambda}+\Lambda_{1}, \quad \alpha=\frac{1}{n h_{d}} \sum_{i=1}^{n} \int_{-\infty}^{\lambda} K_{d}\left(\frac{F_{n h_{r}}(i / n)-u}{h_{d}}\right) d u
$$

We let

$$
\mu_{1}=x_{\lambda}^{2} \quad \mu_{2}=x_{\lambda}
$$

The representation

$$
\begin{aligned}
& \hat{x}_{2, \lambda}-\frac{\mu_{1}}{\mu_{2}}=\frac{\beta-\mu_{1}}{\mu_{2}}-\frac{\mu_{1}}{\mu_{2}^{2}}\left(\alpha-\mu_{2}\right)+ \\
& \quad+O_{p}\left(\left(\beta-\mu_{1}\right)\left(\alpha-\mu_{2}\right)\right)+O_{p}\left(\left(\alpha-\mu_{2}\right)\right)
\end{aligned}
$$

(see[10, p. 327]) and the fact that, as $n \rightarrow \infty$,

$$
\tilde{g}^{2}=\frac{g_{1}^{2}}{\mu_{2}^{2}}+\frac{g_{2}^{2} \mu_{1}^{2}}{\mu_{2}^{4}}-2 \operatorname{cov}\left(\frac{\beta}{\mu_{2}}, \frac{\alpha \mu_{1}}{\mu_{2}^{2}}\right) \sim g^{2}=\frac{\lambda(1-\lambda)}{f^{2}\left(x_{\lambda}\right)}\left\|K_{r}\right\|^{2}
$$

imply the following theorem.

Theorem 4.2. As $n \rightarrow \infty$,

$$
\sqrt{n h_{r}}\left(\hat{x}_{2, \lambda}-x_{\lambda}-b\left(h_{r}, h_{d}\right)\right) \stackrel{d}{\rightarrow} N\left(0, g^{2}\right),
$$

where

$$
\begin{gathered}
b_{1}\left(h_{r}, h_{d}\right)=a_{1, d} h_{d}^{2}+a_{1, r} h_{r}^{2}, \\
a_{1, r}=-\frac{\nu_{r}^{2} x_{\lambda} f^{\prime}\left(x_{\lambda}\right)}{f^{4}\left(x_{\lambda}\right)}, \quad a_{1, d}=\nu_{d}^{2}\left(-\frac{x_{\lambda} f^{\prime}\left(x_{\lambda}\right)}{f^{3}\left(x_{\lambda}\right)}+\frac{1}{f^{2}\left(x_{\lambda)}\right.}\right) .
\end{gathered}
$$

In Lemma 4.7 and Theorem 4.2 there appear the quantities $a_{1, r}$ and $a_{1, d}$ involving the derivatives of the inverse function $F^{-1}(\lambda)$, namely, $\left(F^{-1}\right)^{\prime}(\lambda),\left(F^{-1}\right)^{\prime \prime}(\lambda)$, which are known. As their estimators, we suggest the following statistics,

$$
\hat{c}_{1}=\frac{1}{n h_{d}} \sum_{i=1}^{n} K_{d}\left(\frac{F_{n h_{r}}(i / n)-\lambda}{h_{d}}\right) \quad \text { and } \quad \hat{c}_{2}=-\frac{1}{n h_{d}^{2}} \sum_{i=1}^{n} K_{d}^{\prime}\left(\frac{F_{n h_{r}}(i / n)-\lambda}{h_{d}}\right) .
$$

Arguing as above, one can show that as $n \rightarrow \infty$, they converge in probability to $\left(F^{-1}\right)^{\prime}(\lambda)$ and $\left(F^{-1}\right)^{\prime \prime}(\lambda)$, respectively. Then a consistent estimator for $\hat{b}_{1}\left(h_{r}, h_{d}\right)$ is $\nu_{r}^{2} h_{r}^{2} \hat{c}_{1} \hat{c}_{2}+\nu_{d}^{2} h_{d}^{2}\left(\hat{c}_{2}+\hat{c}_{1}^{2}\right)$.

Theorem 4.2 implies that the dispersion of the limiting distribution of the estimator $\hat{x}_{2, \lambda}$ is the same as for the estimator $\hat{x}_{1, \lambda}$ and this is why we consider the estimator

$$
\hat{x}_{3, \lambda}=\sqrt{\hat{S}_{2, \lambda}-\hat{b}_{1}\left(h_{r}, h_{d}\right)} .
$$

Employing Theorem 3.1, it is easy to obtain the following result.

Theorem 4.3. As $n \rightarrow \infty$,

$$
\sqrt{n h_{r}}\left(\hat{x}_{3, \lambda}-x_{\lambda}\right) \underset{n \rightarrow \infty}{\stackrel{d}{\longrightarrow}} N\left(0, g_{3}^{2}\right)
$$

where

$$
g_{3}^{2}=\frac{\lambda(1-\lambda) x_{\lambda}}{f^{2}\left(x_{\lambda}\right)}\left\|K_{r}\right\|^{2}
$$


Since $0<x_{\lambda}<1$, by Theorem 4.3 we conclude that the limiting dispersion of the estimator $\hat{x}_{3, \lambda}$ is less than that of the estimators $\hat{x}_{1, \lambda}$ and $\hat{x}_{2, \lambda}$.

The constructed estimator $\hat{x}_{3, \lambda}$ was employed to find effective doses for the examples borrowed from book [1] as well as for the Finney's example, see [11, p. 98].

\section{BIBLIOGRAPHY}

1. S.V. Krishtopenko, M.S. Tikhov, E.B. Popova. Dose-effect. Medicina, Moscow. 2008

2. V.M. Kocheganov, M.S. Tikhov. Estimating of effective doses in dose-response relationship // Obozrenie prikl. promyshlen. matem. 2011. V. 18, No. 1. P. 85-86. (in Russian.)

3. H. Dette, N. Neumeyer, K.F. Pilz A note on nonparametric estimation of the effective dose in quantal bioassay // J. Am. Stat. Assoc. 2005. V. 100, No. 470. P. 503-510.

4. I.P. Natanson. Theory of functions of a real variable. Lan', Moscow. 2008. [F. Ungar Pub. Co., New York. 1961.]

5. H. Niederreiter. Random number generation and quasi-Monte Carlo methods. Society for industrial and applied mathematics, Philadelphia, Pensilvania. 1992.

6. E.L. Lehmann. Theory of point estimation. John Wiley \& Sons, New York. 1983.

7. E.L. Lehmann. Theory of point estimation. Nauka, Moscow. 1991.

8. M.S. Tikhov, D.S. Krishtopenko, M.V. Yaroschuk. Estimation of distributions in dose-response relationship for fixed plan of experiment // Statistical methods for estimating and verification of hypotheses. Interuniverisities collection of scientific works. Permskij univer. Perm. 2006. P. 66-77. (in Russian.)

9. H. Cramér. Mathematical methods of statistics. Mir Publisher, Moscow. 1976. [Princeton Univ. Press, Princeton. 1999.]

10. M.S. Tikhov. Statistical estimation on the basis of interval-censored data // Journal Math. Sci. 1974. V. 119, No. 3. P. 321-335.

11. D.J. Finney. Probit Analysis. Cambridge University Press, New York. 1971.

Mikhail Semenovich Tikhov,

Lobachevsky State University of Nizhni Novgorod,

Gagarin avenue, 23

603950, Nizhni Novgorod, Russia

E-mail: tikhovm@mail.ru 